\title{
A Method for the Measurement of Temperature Based on Multisensor Data Fusion
}

\author{
Gaoli Chen ${ }^{1, a}$,Chengfang $\mathrm{Ji}^{1, \mathrm{~b}}$ \\ ${ }^{1}$ Zhengzhou University of Industry Technology, Zhengzhou 450000, China. \\ aamy000@163.com, bjichengfang8897@163.com
}

Keywords: Multi-sensor, temperature acquisition, Data fusion, distribution display method, Parameter estimation.

Abstract. The performance of the boiler heat-treatment is restricted by temperature. At present, it is a technical problem that should be solved immediately for accurately and reliably measuring the boiler temperature of heat-treatment. This paper presents a new method of multisensor data fusion for measuring the temperature based on Bayesian estimation, and gives the data fusion algorithm. The experiment results show that the algorithm is reasonable and reliable, improving the accuracy of the temperature acquisition, and effectively eliminate the error caused by the failure sensor. It can be used in multisensor systems which the measurement data be-long to normal distribution.

\section{Introduction}

The temperature of the boiler is a very important parameter for life and industrial production, especially in the heat, power system, etc[1]. So it is very important to control the boiler temperature effectively. To produce steam boiler equipment and control the temperature in a certain range is the important measures to improve the boiler safety and economy[2].

This paper is main to research temperature acquisition of the boiler, the use of multi-sensor data fusion based on the method of growing detects the temperature of the boiler, excluding measurement outliers, reducing sensor detecting loss of information, more accurate, and more reliable measurement results.

\section{The method of temperature divorced value eliminating}

When a temperature sensor with the boiler sudden interference in the measurement, inevitably produce false even the error of the measured value. If the divorce value of the sensor data into the fusion center, will greatly affect the precision of the fusion. So before the data fusion of multiple sensors must remove divorce value[3,4].

The boiler system adopts eight temperature sensor groups to acquire temperature. When processing divorce value, distributing chart method eliminate divorced[5] value accuracy high, in this paper uses this method. The parameters which reflect the structure of data distribution in the distribution of temperature measuring mainly has the median $T_{M}$, upper quartile $F_{u}$, lower quartile $\mathrm{F}_{1}$ and quartile discrete degree $\mathrm{dF}$ and eliminate points $\rho$. The definition and usage are as follows:

(1) Suppose $N$ the measured value of the temperature sensor $T_{i}$ has been order from small to large be $T_{1} 、 T_{2} T_{3} \ldots \ldots T_{N}$, so $T_{1}$ and $T_{N}$ is respectively called up and down extremum,

(2) Define the median value $\boldsymbol{T}_{M}= \begin{cases}\frac{T}{\frac{N+1}{2}} & (N \text { is odd }) \\ \frac{\mathrm{T}_{\frac{N}{2}}+\mathrm{T}_{\frac{N}{2}+1}}{2} & (N \text { is even })\end{cases}$

(3) Bitmap in the points of upper and lower quartile definition respectively interval range $\left[T_{M}\right.$ $\left.\mathrm{T}_{\mathrm{N}}\right]$ median and range of $\left[\mathrm{T}_{1} \mathrm{~T}_{\mathrm{M}}\right]$ median

(4) Quartile of discrete degree $d F=F_{u}-F_{l}$. If the cognizance and distance is greater than the median $\beta \mathrm{dF}$ is divorced value, so when located in the $\left|T_{i}-T_{M}\right|>\beta d F$, $\mathrm{T}_{\mathrm{i}}$ is invalid data, then get the corresponding invalid points $\rho_{1}=F_{1}-\frac{\beta}{2} d F, \rho_{2}=F_{u}+\frac{\beta}{2} d F \quad$, in this system $\beta=2$. 
The matlab simulation which divorce values for temperature data measured by eight sensors at one moment is shown in figure 1. Due to the distribution of data and the choice of $\mathrm{dF}$ only and position, and has nothing to do with the size of the extreme value point. So using histogram method to obtain consistent sensor data method has robustness.

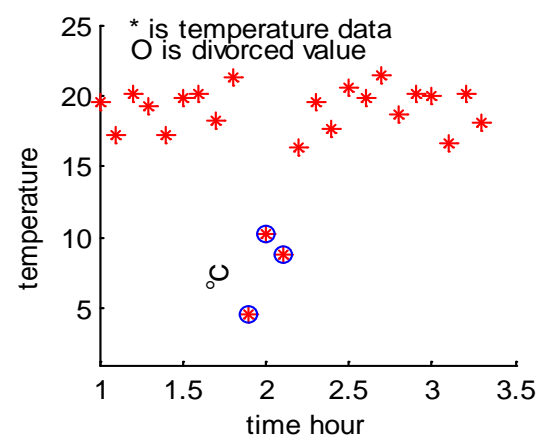

Figure 1 simulation of eliminating temperature divorce values

\section{The multi-sensor data fusion algorithm of temperature acquisition}

In this paper, the data fusion algorithm based on Bayesian estimation is applied. Bayesian estimation is to make each sensor as a Bayesian estimator. It is for each sensor information do compatibility analysis, eliminating those low credibility of error messages. Eight temperature sensor by the transmitter into standard of $0 \sim 5 \mathrm{v}$ voltage signal, to set the voltage sequence is $\mathrm{V}_{1}$ 、 $\mathrm{V}_{2} 、 \ldots \ldots, \mathrm{V}_{\mathrm{n}}$, Because error, the actual measured values(the voltage) is

$$
V_{i}=A_{i}+E_{i} \quad(i=1,2 \ldots \ldots . . .8)
$$

Among $\mathrm{A}_{\mathrm{i}} \mathrm{T}$ - the observation results, $\mathrm{A}_{\mathrm{i}}$ - known constants, $\mathrm{E}_{\mathrm{i}}$-measurement error

The Bayesian estimation theory, get $\mathrm{n}$ optimal estimation for temperature measurement data is $\operatorname{Topt}\left(V_{1}^{\wedge} V_{2} \ldots . . V_{n}\right)=\wedge_{T}^{\max } P\left(T \mid V_{1} V_{2} \ldots . . V_{n}\right)$

By the Bayesian theorem get $P\left(T \mid V_{1} V_{2} \cdots \cdots V_{n}\right)=\frac{P(T) P\left(V_{1} V_{2} \cdots \cdots V_{n} \mid T\right)}{P\left(V_{1} V_{2} \cdots \cdots V_{n}\right)}$

Due to $\mathrm{n}$ observations are independent of each other, there are

$$
P\left(V_{1} V_{2} \ldots \ldots V_{n}^{T}\right)=\prod_{i=1}^{n} P\left(V_{i} \mid T\right)
$$

By type (4)and type (5) get $P\left(T \mid V_{1} V_{2} \cdots \cdots V_{n}\right)=\frac{\prod_{i=1}^{n} P\left(T \mid V_{i}\right) \prod_{i=1}^{n} P\left(V_{i}\right)}{[P(T)]^{n-1} P\left(V_{1} V_{2} \cdots \cdots V_{n}\right)}$

Among $P\left(T \mid V_{1} V_{2} \ldots \ldots V_{n}\right)$ and $\prod_{i=1}^{n} P\left(V_{i}\right)$ has nothing to do with $\mathrm{T}$, in the maximum a posteriori estimation $\mathrm{T}(\mathrm{V})$ can't be considered. So have to $\mathrm{n}$ times value of temperature measurement fusion is $\quad \hat{T}(V)=\frac{\sum_{i=1}^{n} C_{n i}^{-1} V_{i}}{\sum_{i=1}^{n} C_{n i}^{-1}}$

Among $\sum_{i=1}^{n} C_{n i}^{-1}=\frac{C T}{V^{-1}}$ is the two random variables related degree between $\mathrm{T}_{\mathrm{i}}$ and $\mathrm{V}_{\mathrm{i}}$ the covariance. 


\section{The results of temperature experiment and data fusion}

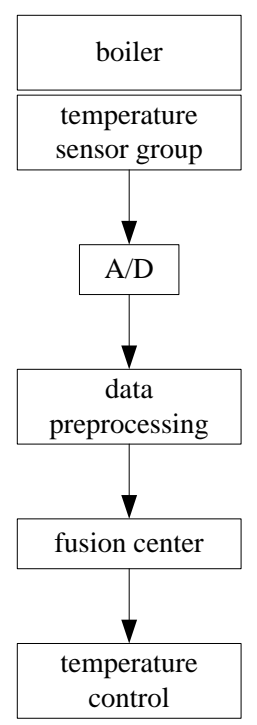

Figure 2 temperature acquisition of the boiler system

The concrete implementation of temperature acquisition in the boiler system is shown in Figure 2: In boiler to place eight temperature sensors in the upper middle and under layer of the special position. These sensors collect the temperature of boiler real-time, and the final temperature of accurate output current environment. Just one moment to eight different location of the temperature sensor collected three groups data, the data are shown in table 1.

table 1 temperature measurements (unit ${ }^{\circ} \mathrm{C}$ )

\begin{tabular}{|c|c|c|c|c|c|c|c|c|c|}
\hline \multicolumn{2}{|c|}{ sensor } & 1 & 2 & 3 & 4 & 5 & 6 & 7 & 8 \\
\hline \multirow{2}{*}{$\begin{array}{l}\text { measured } \\
\text { value }\end{array}$} & 1 & 79.5 & 78.3 & 81.2 & 64.6 & 77.1 & 79.7 & 77.4 & 77.9 \\
\cline { 2 - 10 } & 2 & 79.8 & 79.5 & 80.7 & 70.3 & 77.2 & 81.2 & 78.3 & 77.2 \\
\cline { 2 - 10 } & 3 & 79.6 & 79.9 & 80.9 & 68.8 & 77.7 & 80.5 & 80.0 & 78.1 \\
\hline
\end{tabular}

The temperature of the first group measurements data according to the ascending order is $64.6^{\circ} \mathrm{C} 、 77.1^{\circ} \mathrm{C} 、 77.4^{\circ} \mathrm{C} 、 77.9^{\circ} \mathrm{C} 、 78.3^{\circ} \mathrm{C} 、 79.5^{\circ} \mathrm{C} 、 79.7^{\circ} \mathrm{C}$ and $81.2^{\circ} \mathrm{C}$. The average temperature $\bar{T}=\frac{1}{8} \sum_{i=1}^{8} T_{i}=76.96^{\circ} \mathrm{C}$, the error between the measured value and the actual value is $3.04^{\circ} \mathrm{C}$. By type (1) the median $\mathrm{T}_{\mathrm{M}}=78.9^{\circ} \mathrm{C}$ known, $\mathrm{T}_{4}$ is divorced value, should be removed. By the fusion of (7) to calculate the temperature value is $\hat{T}(V)=78.85^{\circ} \mathrm{C}$, the error between the measured value and the actual value is $1.15^{\circ} \mathrm{C}$. The results show that the fusion algorithm based on Bayesian estimation not only improve the precision of temperature acquisition, but also can effectively eliminate the error caused by sensor failure.

\section{Summary}

This article using distribution display method to eliminate divorced values and multi-sensor data fusion algorithm based on Bayesian parameter estimation is applied to the boiler temperature acquisition system research. Bayesian parameter estimation of multi-sensor data fusion algorithm, and a small amount of calculation and programming is simple, in the case of other conditions constant improve the reliability of the measurement data. 


\section{References:}

[1] R Luo, M Kay. Multisensor integration and fusion: issues and approaches[J]. SPIE,1988,931:195-202.

[2] Xiangqian Chu,Wu Zhu. Multi-sensor Data Fusion of Temperature in a Greenhouse[J]. Journal of Agricultural Mechanization Research,2008 (4) : 180-181.

[3] Gaoli Chen, Liguo Tian,Meng Li and Zhiqi Liu. Research on the Intelligent Plant Growth System Temperature Acquisitio Based n on the Data Fusion. 2013(ICICEE 2013):906-909.

[4] L.Wald. Some terms of reference in data fusion[J]. IEEE Transactions on Geoscience and Remote Sensing,1999,37(3):1190-1193.

[5] P. K. Varshney, "Multisensor data fusion” [J]. Electronics \& Communication Engineering Journal, December 1997, 245-253. 